\section{Hat Ihr Kind Asthma?}

B ei der Erhebung von Daten zur Asthma-Prävalenz bei Kindern greifen Epidemiologen gern auf die Angaben der Eltern zurück - falsche Ergebnisse sind so vielleicht vorprogrammiert, warnt jetzt eine US-Studie. Mit Hilfe des Medical Expenditure Panel Survey war es möglich, den Medikamentenverbrauch von 10.404 kalifornischen Kindern im Alter zwischen 0 und 17 Jahren zu dokumentieren, gleichzeitig wurden die Eltern zur Gesundheit

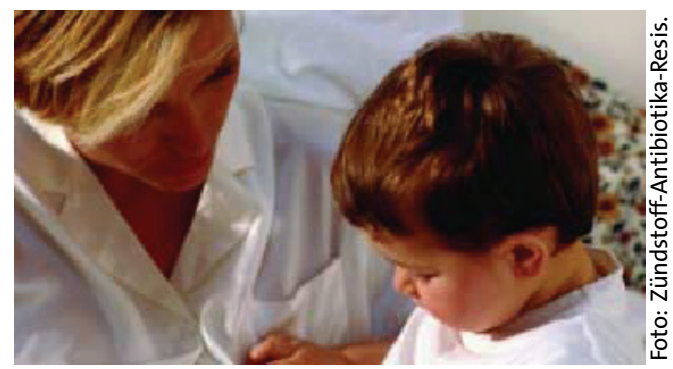

ihres Nachwuchses befragt. Es zeigte sich, dass $41 \%$ der Eltern, die regelmäBig inhalative Glukokortikoide oder Mastzellstabilisatoren für ihr Kind in der Apotheke holten, der Meinung waren, ihr Spross habe kein Asthma. nbr

Roberts EM. Arch Pediatr Adolesc Med

2003; 157: 449-55

\section{Gefährlicher Kuss}

$\mathrm{N}$ ach dem Gutenachtkuss ihres Freundes erlitt eine 20-jährige Amerikanerin beinahe einen tödlichen anaphylaktischen Schock. Die lebensbedrohliche Atemnot konnte gerade noch rechtzeitig stationär beherrscht werden. Der Grund für den Anfall: Ihr Freund hatte etwa eine Stunde vor dem zärtlichen Überfall Shrimps gegessen. Die Patientin wusste zwar von ihrer Allergie auf Krustentiere, hatte aber nicht mit einer Reaktion auf die MeeresfrüchteMahlzeit ihres Freundes gerechnet. uls

Steensma DP. Mayo Clin Proc 2003; 78 : 221-2

\title{
Patienten bevorzugen Fachchinesisch
}

W er sich bemüht, bei der Erklärung eines Krankheitsbildes medizinische Fachtermini zu vermeiden, handelt damit nicht unbedingt im Sinn des Patienten. Das behaupten britische Mediziner, die 900 Hausarztpatienten gebeten hatten, Diagnosen mit Fachausdrücken beziehungsweise mit den entsprechenden Übersetzungen in die Alltagssprache zu bewerten. Das überraschende Ergebnis: Die Patienten bevorzugten eine Gastroenteritis gegenüber einer Magenverstimmung und hatten lieber eine Tonsillitis statt Halsschmerzen. Durch die Verwendung der medizinischen Termini fühlten sie sich mit ihren Beschwerden ernst genommen und machten sich größere Hoffnungen auf eine Krankschreibung. Sie schätzten außerdem die Fähigkeiten ihres Arztes höher ein und waren insgesamt zufriedener mit dem Praxisbesuch.

$D E$

Ogden J et al. Fam Pract 2003; 20: 248-53

\section{Schützt BCG-Impfung vor Atopie?}

$B_{n}^{i}$ 1990 erhielten in Grönland alle Neugeborenen eine BCG-Vakzination zum Schutz vor Tuberkulose, danach sahen die offiziellen Empfehlungen die Impfung nicht mehr vor. Ideale Voraussetzungen also, um die These zu überprüfen, wonach die BCG-Impfung eine Immunreaktion vom TH1-Typ induziert und damit gleichzeitig die Expression von TH2-Zytokinen unter- drückt - letztlich also vor atopischen Erkrankungen schützt. Dänische Wissenschaftler bestimmten dazu bei 1.686 Kindern die spezifischen IgE-Antikörper gegen typische Aeroallergene. Enttäuschendes Ergebnis: Die BCG-Impfung verringerte nicht, wie gehofft, das Risiko einer Atopie.

$n b r$

Krause TG et al. JAMA 2003; 289: 1012-5

\section{Gegenwind für Glimmstängel-Gegner}

D assivrauchen fördert die Entstehung von Herz-Kreislauf-Krankheiten und erhöht das Risiko, an Lungenkrebs zu erkranken. Dieses Wissen zählt schon fast zum Allgemeingut und wird doch von einer aktuellen prospektiven Kohortenstudie aus Kalifornien komplett in Frage gestellt. Epidemiologen hatten von 1959 bis 1998 die Daten von 36.561 Paaren erhoben, von denen ein Partner Raucher, der andere Nichtraucher war. Das Risiko, an Lungenkrebs, koronaren Herzkrankheiten oder COPD zu erkranken, war bei den Rauchern wie zu erwarten deutlich erhöht, für die passiv rauchenden Partner war dagegen dieser Zusammenhang überhaupt nicht gegeben. Die hohe Teilnehmerzahl und die lange Beobachtungszeit verleihen der Untersuchung einiges an Gewicht, es sollte aber auch nicht verschwiegen werden, dass die Tabakindustrie einer der Hauptsponsoren der Studie war.

Enstrom JE et al. BMJ 2003; 326: 1057-61

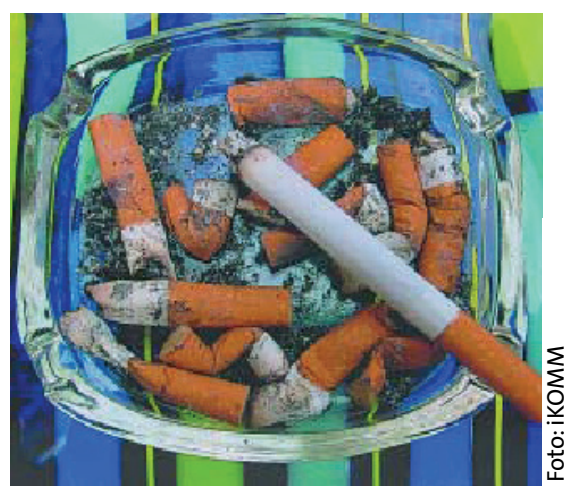

